\title{
Homotopy Perturbation Method for Brachistochrone Problem
}

\author{
Parivash Shams Derakhsh", Parisa Shams Derakhsh \\ Department of Mathematics, Faculty of Science University of Guilan, P.O. Box41335-1914, P.C. 41938, Rasht, Iran \\ *Corresponding Author: shams9077@yahoo.com
}

Copyright (c) 2013 Horizon Research Publishing All rights reserved.

\begin{abstract}
The most famous classical variational principle is the so-called Brachistochrone problem. In this work, Homotopy perturbation method (HPM) is applied to the Brachistochrone problem that arises invariational problems. The results reveal the efficiency and the accuracy of the proposed method. Homotopy perturbation method yields solutions in convergent series forms with easy computation.
\end{abstract}

Keywords Brachistochrone Problem, Homotopy Perturbation Method, Nonlinear Equations

\section{Introduction}

Minimization problems that can be analyzed by the calculus of variations serve to characterize the equilibrium configurations of almost all continuous physical systems, ranging through elasticity, solid and fluid mechanics, electro-magnetism, gravitation, quantum mechanics, string theory, and many other topics. Some of geometrical configurations such as minimal surfaces, can be conveniently formulated as optimization problems. Moreover, numerical approximations to the equilibrium solutions of such boundary value problems are based on a nonlinear finite element approach that reduced the infinite dimensional minimization problem to a finite-dimensional one, to which we can apply the optimization techniques.

The compound Greek word "Brachistochrone" means "minimal time". An experimenter lets a bead slide down a wire that connects two fixed points. The goal is to shape the wire in such a way that, starting from rest, the bead slides from one end to the other in minimal time. Naive guesses for the wire's optimal shape, including a straight line, a parabola, a circular arc, or even a catenaries are wrong. One can do better through a careful analysis of the associated variational problem. The Brachistochrone problem was originally posed by the Swiss mathematician Johann Bernoulli in 1696, and served as an inspiration for much subsequent development of this subject.

Homotopy perturbation method (HPM) is a useful and a powerful method for solving linear and nonlinear differential equations, partial differential equations, and integral equations. In this method the solution is considered as the sum of an infinite series which usually convergence rapidly to an accurate solutions.

Many researchers have been successfully applied Homotopy perturbation method to various nonlinear problems in science and engineering, such as the viscous flows of non-Newtonian fluids [1], the $\mathrm{KdV}$-type equations [2], finance problems [3], nonlinear optimal control problems [4- 5] and some others.

\section{Homotopy Perturbation Method (HPM)}

Let us consider the following non-linear functional equation:

$$
\begin{aligned}
\mathrm{A}(\mathrm{u})-\mathrm{f}(\mathrm{r})=0, & \mathrm{r} \in \Omega, \\
\mathrm{B}\left(\mathrm{u}, \frac{\partial u}{\partial n}\right)=0, & \mathrm{r} \in \Gamma .
\end{aligned}
$$

Where $\Gamma$ is boundary of the domain $\Omega$, A is a functional operator and $\mathrm{f}$ is a known analytic function. The operator $\mathrm{A}$ can be decomposed into a linear and invertible part $\mathrm{L}$, and a non-linear part N. Hence Eq. (1) can be written as the following form:

$$
L(u)+N(u)-f(r)=0 .
$$

Using Homotopy technique, we construct a Homotopy $\mathrm{v}(\mathrm{r}, \mathrm{p}): \Omega \times[0,1] \rightarrow \mathrm{R}$, which satisfies

$$
\mathrm{H}(\mathrm{r}, \mathrm{p})=(1-\mathrm{p})\left[\mathrm{L}(\mathrm{v})-\mathrm{L}\left(\mathrm{u}_{0}\right)\right]+\mathrm{p}[\mathrm{A}(\mathrm{v})-\mathrm{f}(\mathrm{r})]=0 .
$$

Or

$$
\mathrm{H}(\mathrm{r}, \mathrm{p})=\mathrm{L}(\mathrm{v})-\mathrm{L}\left(\mathrm{u}_{0}\right)+\mathrm{pL}\left(\mathrm{u}_{0}\right)+\mathrm{p}[\mathrm{N}(\mathrm{v})-\mathrm{f}(\mathrm{r})]=0 .
$$

Where $p \in[0,1]$ is an embedding parameter and $\mathrm{u}_{0}$ is an initial approximation to the solution of Eq. (1) which satisfies the boundary conditions. Obviously, from Eq. (4) we have

$$
\mathrm{H}(\mathrm{v}, 0)=\mathrm{L}(\mathrm{v})-\mathrm{L}\left(\mathrm{u}_{0}\right)=0, \mathrm{H}(\mathrm{v}, 1)=\mathrm{A}(\mathrm{v})-\mathrm{f}(\mathrm{v})=0 .(6)
$$


Let's consider the solution of Eq. (5) as a power series over p, as follows:

$$
\mathrm{v}=\mathrm{v}_{0}+\mathrm{pv}_{1}+\mathrm{p}^{2} \mathrm{v}_{2}+\ldots \ldots,
$$

The approximate solutions of Eq.(1) can be obtained by letting $\mathrm{p} \rightarrow 1$

$$
\mathrm{u}=\lim _{p \rightarrow 1} v=v_{0}+v_{1}+v_{2}+\cdots
$$

Substituting this series into Eq. (5), leads to

$$
\mathrm{L}\left(u_{0}\right)=p\left[f(r)-L\left(u_{0}\right)-N\left(\sum_{i=0}^{\infty} v_{i} p^{i}\right)\right]
$$

Putting (7) into (8), and comparing the coefficients of the terms with the identical powers of $p$

$$
\begin{array}{rr}
\mathrm{P}^{0}: \mathrm{L}\left(\mathrm{v}_{0}\right)-\mathrm{L}\left(\mathrm{u}_{0}\right)=0, \\
\mathrm{P}^{1}: L\left(v_{1}\right)=f(r)-N\left(\mathrm{u}_{0}\right)-\mathrm{L}\left(\mathrm{u}_{0}\right), & \mathrm{v}_{1}(\mathrm{x}, 0)=0, \\
\mathrm{p}^{2}: \mathrm{L}\left(v_{2}\right)=-\mathrm{N}\left(v_{1}\right), & \mathrm{v}_{2}(\mathrm{x}, 0)=0,
\end{array}
$$

So;

$$
\begin{array}{r}
\mathrm{v}_{0}=\mathrm{u}_{0}, \\
v_{1}=\mathrm{L}^{-1}(\mathrm{f}(\mathrm{r}))-\mathrm{L}^{-1}\left(\mathrm{~N}\left(\mathrm{u}_{0}\right)\right)-\mathrm{L}^{-1}\left(\mathrm{~L}\left(\mathrm{u}_{0}\right)\right), \\
\mathrm{v}_{2}=-\mathrm{N}\left(\mathrm{v}_{1}\right), \\
\vdots \\
\mathrm{u}_{\mathrm{HPM}}=\mathrm{L}^{-1}(\mathrm{f}(\mathrm{r}))-\mathrm{L}^{-1}\left(\mathrm{~N}\left(\mathrm{u}_{0}\right)\right)-\mathrm{N}\left(\mathrm{v}_{1}\right)+\ldots .
\end{array}
$$

\section{The Brachistochrone Problem}

We take, without loss of generality, the starting point of the bead to be at the origin $\mathrm{a}=(0,0)$. The wire will bend downwards, and so, to avoid distracting minus signs in the subsequent formulae, we take the vertical y axis to point downwards. The shape of the wire will be given by the graph of a function $y=u(x) \geq 0$. The end point $b=(b, \beta)$. The end point $b=(b, \beta)$ is assumed to lie below and $b>0, \beta>0$. The set-up is sketched in Figure 1.

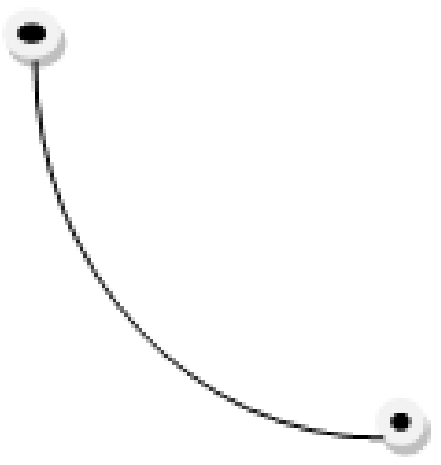

Figure1. The Brachistrochrone Problem.

Let us concentrate on the simplest class of Variational problems, in which the unknown is a continuously differentiable scalar function, and the functional to be minimized depends upon at most its first derivative. The basic minimization problem, then, is to determine a suitable function $\mathrm{y}=\mathrm{u}(\mathrm{x}) \in \mathrm{C}^{1}[\mathrm{a}, \mathrm{b}]$ that minimizes the objective functional

$$
\mathrm{J}[\mathrm{u}]=\int_{a}^{b} L(x, u, u) d x
$$

The integrand is known as the Lagrangian for the Variational problem, in honor of Lagrange, one of the main founders of the subject.

Integrating from start to finish, we conclude that the total travel time along the curve is equal to

$$
T[u]=\int_{0}^{T} d t=\int_{a}^{b} \frac{d t}{d x} d x=\int_{a}^{b} \frac{\sqrt{1+\left(u\left(\mathbb{Q}_{x}\right)\right)^{2}}}{c(x, u(x))} d x .
$$

Where

$$
\mathrm{C}(\mathrm{x}, \mathrm{u}(\mathrm{x}))=\frac{d s}{d t}=1+(u(X))^{2} \frac{d x}{d t} .
$$

To mathematically formulate the problem, the first step is to find the formula for the transit time of the bead sliding along the wire. Arguing as in our derivation of the optics functional (11), if $\mathrm{v}(\mathrm{x})$ denotes the instantaneous speed of descent of the bead when it reaches position $(x, u(x))$, then the total travel time is

$$
\mathrm{T}[\mathrm{u}]=\int_{0}^{l} \frac{d s}{v}=\int_{0}^{b} \frac{\sqrt{1+(u(X))^{2}}}{v} \mathrm{dx} .
$$

Where $\ell$ is the overall length of the wire. We shall use conservation of energy to determine a formula for the speed $\mathrm{v}$ as a function of the position along the wire. The kinetic energy of the bead is $\frac{1}{2} \mathrm{mv}^{2}$, where $\mathrm{m}$ is its mass.

On the other hand, due to our sign convention, the potential energy of the bead when it is at height $y=u(x)$ is $\mathrm{mg} u(\mathrm{x})$, where $\mathrm{g}$ the gravitational force, and we take the initial height as the zero potential energy level. The bead is initially at rest, with 0 kinetic energy and 0 potential energy. Assuming that frictional forces are negligible, conservation of energy implies that the total energy must remain equal to 0 , and hence

$$
\frac{1}{2} m v^{2}-\mathrm{mg} \mathrm{u}=0 .
$$

We can solve this equation to determine the bead's speed as a function of its height

$$
\mathrm{v}=\sqrt{2 \mathrm{gu}} .
$$

Substituting this expression into (12), we conclude that the shape $y=u(x)$ of the wire is obtained by minimizing the functional

$$
\mathrm{T}[\mathrm{u}]=\int_{0}^{b} \sqrt{\frac{1+(u)^{2}}{2 g u}} \mathrm{dx}
$$

subject to the boundary conditions

$$
\mathrm{u}(0)=0, \quad \mathrm{u}(\mathrm{b})=\beta .
$$

The associated Lagrangian is

$$
\mathrm{L}(\mathrm{x}, \mathrm{u}, \mathrm{p})=\sqrt{\frac{1+p^{2}}{u}}
$$


where we omit an irrelevant factor of $\sqrt{2 \mathrm{~g}}$, We compute

$$
\frac{\partial L}{\partial u}=\frac{-\sqrt{1+p^{2}}}{2 u^{\frac{3}{2}}}, \quad \frac{\partial L}{\partial p}=\frac{p}{\sqrt{u\left(1+p^{2}\right)}} .
$$

Thus, the minimizing functions solve the nonlinear second order ordinary differential equation

$$
2 u u^{\prime \prime}+\left(u^{\prime}\right)^{2}+1=0
$$

This equation has been solved by Hamiltonian function, and so the solution to the Euler-Lagrange equation are curves parameterized by

$$
\mathrm{x}=\mathrm{r}(\theta-\sin \theta), \quad \mathrm{u}=\mathrm{r}(1-\cos \theta) .
$$

The minimization curve is known as a cycloid, can be visualized as the curve traced by a point sitting on the edge of a rolling wheel of radius $r$, as plotted in Figure 2.

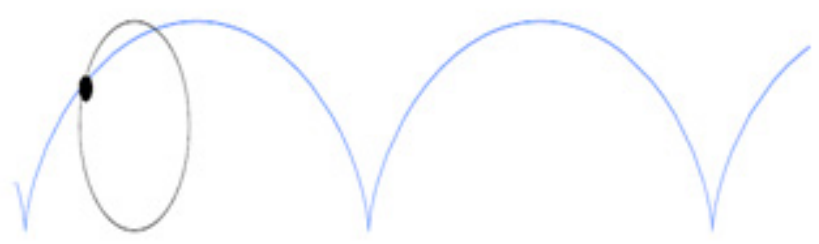

Figure2. A Cycloid

\section{The Structure of HPM Algorithm}

In this section, the program has been provided with Mathematica 6 according to the following algorithm, where $\epsilon$ is a given positive value.

\section{Algorithm}

Step1. Set $\mathrm{n} \leftarrow 0$.

Step 2. Calculate the recursive relations (9).

Step3. If $\left|u_{n+1}-u_{n}\right|<\epsilon$ then go to step 4 else $\mathrm{n} \leftarrow \mathrm{n}+1$ and go to step 2 .

Step 4. Print $\mathrm{u}(\mathrm{x}, \mathrm{t})=\sum_{i=0}^{n} u_{i}(\mathrm{x}, \mathrm{t})$ as the approximate of the exact solution.

Lemma 4.1. The computational complexity of the HPM is $\mathrm{O}\left(n^{2}\right)$.

Proof:

In step 2,

$\mathrm{u}_{0}: 2$,

$\mathrm{u}_{1}: 10$,

$\mathrm{u}_{2}: 20$,

$\mathrm{u}_{\mathrm{n}+1}: 10 \mathrm{n}+10, \quad \mathrm{n} \geq 0$.

In step 4 , the total number of the computations is equal to $\sum_{i=0}^{n} u_{i}(x, t)=O\left(n^{2}\right)$.

\section{Applying HPM to Solve Brachistochrone Problem}

Now we try to solve the minimizing functions with HPM.

$$
2 u u^{\text {回米 }}\left(u^{2}\right)^{2}+1=0, \quad \mathrm{u}\left(x, \frac{\pi}{2}\right)=\frac{2 x}{(\pi-2)}
$$

Suppose the solution of Eq.(16) has the following form

$$
\mathrm{V}=\mathrm{v}_{0}+\mathrm{pv}_{1}+\mathrm{p}^{2} \mathrm{v}_{2}+\ldots .
$$

By Substituting Eq.(17) into Eq.(16) and equating the terms with identical powers of $p$, according to the Homotopy perturbation technique, we construct the following Homotopy

$$
\begin{array}{r}
(1-\mathrm{p})\left(\frac{\partial^{2} \mathrm{v}}{\partial \mathrm{x}^{2}}-\frac{\partial^{2} \mathrm{u}_{0}}{\partial \mathrm{x}^{2}}\right)+\mathrm{p}\left(\frac{\partial^{2}\left(\mathrm{v}_{0}+\mathrm{pv}_{1}+\mathrm{p}^{2} \mathrm{v}_{2}+\cdots\right.}{\partial \mathrm{x}^{2}}\right)+ \\
\frac{\left(\left(\mathrm{v}_{0}+\mathrm{p} \mathrm{v}_{1}+\mathrm{p}^{2} \mathrm{v}_{2}+\cdots\right)\right)^{2}}{2\left(\mathrm{v}_{0}+\mathrm{pv}_{1}+\mathrm{p}^{2} \mathrm{v}_{2}+\cdots\right)}+\frac{1}{2\left(\mathrm{v}_{0}+\mathrm{pv}_{1}+\mathrm{p}^{2} \mathrm{v}_{2}+\cdots\right)}=0 . \\
\mathrm{P}^{0}: \frac{\partial^{2} \mathrm{v}_{0}}{\partial \mathrm{x}^{2}}-\frac{\partial^{2} \mathrm{u}_{0}}{\partial \mathrm{x}^{2}}=0, \quad \mathrm{v}_{0}\left(\mathrm{x}, \frac{\pi}{2}\right)=\frac{2 \mathrm{x}}{(\pi-2)}, \\
\mathrm{P}^{1}: \frac{\partial^{2} \mathrm{v}_{1}}{\partial \mathrm{x}^{2}}+\frac{\partial^{2} \mathrm{u}_{0}}{\partial \mathrm{x}^{2}}+\frac{1}{2} \mathrm{v}_{0}+\frac{1}{2 \mathrm{v}_{0}}=0, \quad \mathrm{v}_{1}\left(\mathrm{x}, \frac{\pi}{2}\right)=0,(18) \\
\mathrm{P}^{2}: \frac{\partial^{2} \mathrm{v}_{2}}{\partial \mathrm{x}^{2}}+\frac{1}{2}\left(\left(2 \mathrm{v}_{1}^{\mathbb{Q}}-\mathrm{v}_{1}\right)-\frac{\mathrm{v}_{1}}{\mathrm{v}_{0}^{2}}\right)=0, \quad \mathrm{v}_{2}\left(\mathrm{x}, \frac{\pi}{2}\right)=0 .
\end{array}
$$

Successive solutions of Eqs.(18) yield to

$$
\begin{array}{r}
\mathrm{V}_{0}=\frac{2 x}{(\pi-2)}, \\
\mathrm{V}_{1}=\frac{-x^{3}}{6(\pi-2)}-\frac{(\pi-2)}{2}(\ln (\mathrm{x})-\mathrm{x}),
\end{array}
$$$$
\mathrm{V}_{2}=\frac{x^{3}}{6(\pi-2)}-\frac{(\pi-2)}{4} \mathrm{x}^{2}+\frac{(\pi-2)}{2}(\ln (\mathrm{x})-\mathrm{x})-\frac{x^{4}}{48(\pi-2)} \text {, }
$$

Therefore, an approximation to the solution will be as the following:

$$
\begin{array}{r}
\mathrm{V}(\mathrm{x}) \approx \frac{2 x}{(\pi-2)}+\frac{-x^{3}}{6(\pi-2)}-\frac{(\pi-2)}{2}(\ln (\mathrm{x})-\mathrm{x})+\frac{x^{3}}{6(\pi-2)}-\frac{(\pi-2)}{4} \\
\mathrm{x}^{2}+\frac{(\pi-2)}{2}(\ln (\mathrm{x})-\mathrm{x})-\frac{x^{4}}{48(\pi-2)} .
\end{array}
$$

Since Homotopy perturbation method has been led to an exact solution comparison in examples of the results in fig3.

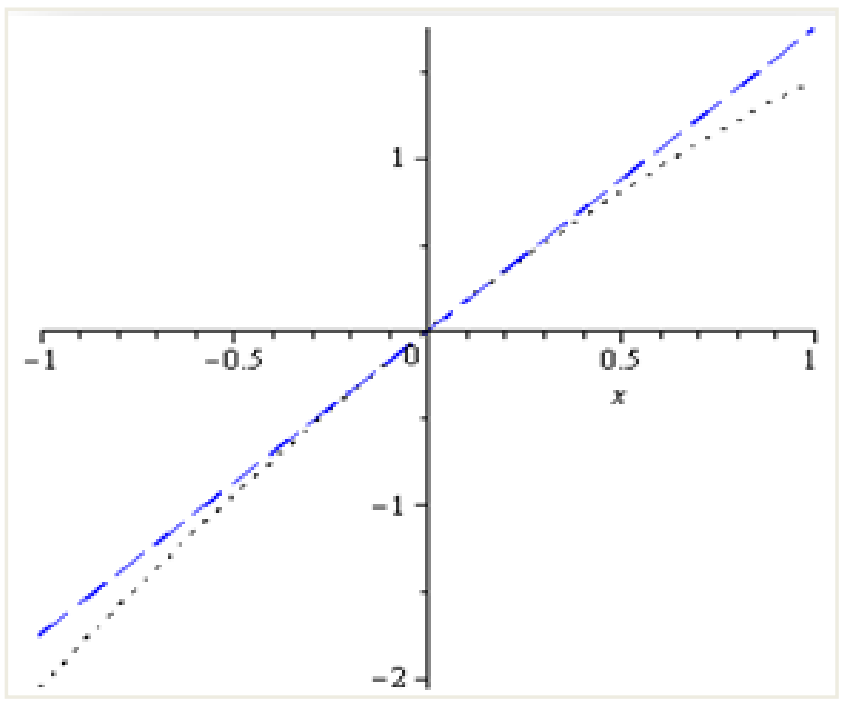

Figure 3. plots of uexact, uHPM 
Table 1. Numerical results for solution of Eq.(16)

\begin{tabular}{llll}
\hline \multicolumn{1}{c}{$\mathrm{x}$} & $\mathrm{U}_{\mathrm{HPM}}$ & $\mathrm{U}_{\text {eract }}$ & $\mathrm{e}\left(\mathrm{U}_{\mathrm{HPM}}\right)$ \\
\hline $10^{-1}$ & $17258 \times 10^{-5}$ & $17543 \times 10^{-5}$ & $2 \times 10^{-3}$ \\
$2 \times 10^{-1}$ & $33944 \times 10^{-5}$ & $35087 \times 10^{-5}$ & $10^{-2}$ \\
$3 \times 10^{-1}$ & $50051 \times 10^{-5}$ & $52631 \times 10^{-5}$ & $2 \times 10^{-2}$ \\
$4 \times 10^{-1}$ & $65568 \times 10^{-5}$ & $70175 \times 10^{-5}$ & $4 \times 10^{-2}$ \\
$10^{-2}$ & $1751 \times 10^{-5}$ & $1754 \times 10^{-5}$ & $2 \times 10^{-5}$ \\
$2 \times 10^{-2}$ & $3497 \times 10^{-5}$ & $3508 \times 10^{-5}$ & $10^{-4}$ \\
$3 \times 10^{-2}$ & $5237 \times 10^{-5}$ & $5263 \times 10^{-5}$ & $2 \times 10^{-4}$ \\
$4 \times 10^{-2}$ & $6971 \times 10^{-5}$ & $7017 \times 10^{-5}$ & $4 \times 10^{-4}$ \\
$10^{-3}$ & $17541 \times 10^{-7}$ & $17543 \times 10^{-7}$ & $2 \times 10^{-7}$ \\
$2 \times 10^{-3}$ & $3507 \times 10^{-6}$ & $3508 \times 10^{-6}$ & $10^{-6}$ \\
$3 \times 10^{-3}$ & $5260 \times 10^{-7}$ & $5263 \times 10^{-7}$ & $2 \times 10^{-6}$ \\
$4 \times 10^{-3}$ & $701298 \times 10^{-8}$ & $701754 \times 10^{-8}$ & $4 \times 10^{-6}$ \\
$10^{-4}$ & $17 \times 10^{-10}$ & $178 \times 10^{-10}$ & $2 \times 10^{-9}$ \\
$2 \times 10^{-4}$ & $3508657 \times 10^{-10}$ & $3508771 \times 10^{-10}$ & $10^{-8}$ \\
$3 \times 10^{-4}$ & $52629 \times 10^{-9}$ & $526315 \times 10^{-9}$ & $2 \times 10^{-8}$ \\
$4 \times 10^{-4}$ & $701708 \times 10^{-9}$ & $701754 \times 10^{-9}$ & $4 \times 10^{-8}$ \\
& & & \\
\hline
\end{tabular}

\section{Conclusions}

In this article, we have applied Homotopy perturbation method for solving Brachistochrone Problem. The approximate solutions obtained by Homotopy perturbation method are compared with the exact solutions. The results show that the error of the method is negligible for $\quad-0.4<\mathrm{x}$
$<0.4$. The results show that the Homotopy perturbation method is a powerful mathematical tool for solving nonlinear ordinary differential equations, which appears in mathematical modeling of different phenomena.

\section{REFERENCES}

[1] T. Hayat, T. Jived and M. Said, "Analytic Solution for Rotating Flow and Heat Transfer Analysis of a Third- Grade Fluid," Acta Mechanical, Vol. 191, No. 3pp. 219-229.

[2] S. Abbas bandy, "Soliton Solutions for the 5th-Order KdV Equation with the Homotopy Analysis Method," Nonlinear Dynamics, Vol. 51, No. 1-2, 2008, pp. 83-87.

[3] S. P. Zhu, "An Exact and Explicit Solution for the Valuation of American Put Options," Quantitative Finance, Vol.6, No. 3, 2006, pp. 229-242.

[4] Biazar, J., Eslami, M., Ghazvini, H., 2007. Homotopy perturbation method for systems of partial differential equations. International Journal of Nonlinear Science and Numerical Simulation 8 (3), 411-416.

[5] S. Efate and H. SaberioNick, "Analytic-Approximate Solution for a Class of Nonlinear Optimal Control Problems by Homotopy Analysis Method of Mathematics, in press. 